\title{
Quantum Field Theory: Where We Are
}

\author{
Klaus Fredenhagen ${ }^{1}$, Karl-Henning Rehren ${ }^{2}$, and Erhard Seiler ${ }^{3}$ \\ 1 II. Institut für Theoretische Physik, Universität Hamburg, \\ 22761 Hamburg, Germany \\ klaus.fredenhagen@desy.de \\ 2 Institut für Theoretische Physik, Universität Göttingen, \\ 37077 Göttingen, Germany \\ rehren@theorie.physik.uni-goe.de \\ 3 Max-Planck-Institut für Physik, \\ 80805 München, Germany \\ ehs@mppmu .mpg.de
}

Summary. We comment on the present status, the concepts and their limitations, and the successes and open problems of the various approaches to a relativistic quantum theory of elementary particles, with a hindsight to questions concerning quantum gravity and string theory.

\section{Introduction}

Quantum field theory aims at a synthesis of quantum physics with the principles of classical field theory, in particular the principle of locality. Its main

Contribution to: An Assessment of Current Paradigms in the Physics of Fundamental Phenomena, to be published by Springer Verlag (2006). 
realm is the theory of elementary particles where it led to a far reaching understanding of the structure of physics at subatomic scales with an often amazingly good agreement between theoretical predictions and experiments. Typical observables in QFT are current densities or energy flow densities which correspond to what is measured in particle physics detectors. The original aim of QFT was to compute expectation values and correlation functions of the observables, and to derive scattering cross sections in high-energy physics. In the course of development, QFT has widened its scope, notably towards the inclusion of gravitational interactions.

Quantum Field Theory rests on two complementary pillars. The first is its broad arsenal of powerful modelling methods, both perturbative and constructive. These methods are based on quantization of classical interactions and the gauge principle, and have been tremendously successful especially for the modelling of all the interactions of the Standard Model of elementary particles. The perturbative treatment of the Standard Model and its renormalization, as well as lattice approximations of Quantum Chromodynamics (QCD), give enormous confidence into the basic correctness of our present understanding of quantum interactions. (For the impressive phenomenological support for the Standard Model, we refer to Dosch's contribution to this volume.) Despite these successes, however, establishing the Standard Model (or part of it) as a mathematically complete and consistent quantum field theory remains an unsettled challenge.

The second pillar of QFT are axiomatic approaches, putting the theory on a firm conceptual ground, which have been developped in order to understand the intrinsic features of a consistent QFT, irrespective of its construction. In these approaches, the focus is set on the fundamental physical principles which any QFT should obey, and their axiomatic formulation in terms of the observable features of a theory is addressed.

In fact, several such axiomatic approaches, which have been shown to be partially but not completely equivalent, are pursued. None of them indicates a necessary failure or inconsistency of the framework of QFT. (Of course, this does not mean that a realistic QFT should not include new Physics, say at the Planck scale, cf. Sect. 8.)

\section{Axiomatic Approaches to QFT}

Axiomatic QFT relies on the fact that the fundamental principles which every quantum field theoretical model should satisfy are very restrictive. On the one hand this is a great obstacle for the construction of models, on the other hand it allows to derive a lot of structural properties which a QFT necessarily has. They often can be tested experimentally, and they provide a criterion whether a construction of a model is acceptable.

The main principles are: 
- the superposition principle for quantum states, and the probabilistic interpretation of expectation values. These two principles together are implemented by the requirement that the state space is a Hilbert space, equipped with a positive definite inner product.

- the locality (or causality) principle. This principle expresses the absence of acausal influences. It requires the commutativity of quantum observables localized at acausal separation (and is expected to be warranted in the perturbative approach if the action functional is a local function of the fields).

In addition, one may (and usually does) require

- covariance under spacetime symmetries (in particular, Lorentz invariance of the dynamics), and

- stability properties, such as the existence of a ground state (vacuum) or of thermal equilibrium states.

The critical discussion of these principles themselves ("axioms") is, of course, itself an issue of the axiomatic approaches. For a review, see [1]. Various axiomatic approaches (Wightman QFT, Euclidean QFT, Algebraic QFT) may differ in the technical way the principles are formulated. Several theorems establishing (partial) equivalences among these approaches have been established, such as the Osterwalder-Schrader reconstruction theorem [2] stating the precise prerequisites for the invertibility of the passage from real time QFT to Euclidean QFT ("Wick rotation"), or the possibility to recover local fields from local algebras [3].

In the Wightman formulation, one postulates the existence of fields as operator-valued distributions defined on a common dense domain within a Hilbert space. The field operators should commute at space-like distance and satisfy a linear transformation law under the adjoint action of a unitary representation of the Poincaré group. Moreover, there should be a unique Poincaré invariant vacuum state which is a ground state for the energy operator. The assumption of local commutativity may be relaxed admitting anticommutativity for fermionic fields. One may also relax the assumption of the vacuum vector, retaining only the positivity of the energy (unless one is interested in thermal states) in order to describe charged states; in the algebraic approach, such theories are most advantageously regarded as different representations (superselection sectors) of the same field algebra, originally defined in the vacuum representation, see below.

Due to the restrictive character of these principles, they typically are violated in intermediate steps of approximation schemes. One often has to introduce auxiliary fields without a direct physical meaning as observables.

As an illustration, consider the Dirac equation featuring a charged electron field coupled to the electromagnetic field:

$$
i \gamma^{\mu}\left(\partial_{\mu}+i e A_{\mu}\right) \psi=m \psi .
$$


The Fermi field $\psi$ satisfies anti-commutation relations and can therefore not be an observable field strength subject to causality. The vector potential $A_{\mu}$ is already in the classical theory not an observable. Related to the gauge arbitrariness, the vector field cannot be covariantly quantized on a Hilbert space with a probabilistic interpretation. (Other problems related with the promotion to QFT of classical field products, appearing in evolution equations such as (1), will be considered later.)

The general principles can therefore not be applied to the objects of basic relations such as (1). The principles rather apply to the physical sector of the theory where the typical fields are current and stress-energy densities or electromagnetic fields, such as

$$
j^{\mu}=\bar{\psi} \gamma^{\mu} \psi, \quad T^{\mu \nu}=T^{\mu \nu}(\psi, A), \quad F_{\mu \nu}=\partial_{\mu} A_{\nu}-\partial_{\nu} A_{\mu} .
$$

These fields, corresponding to observable quantities, should be well-defined in a QFT, admitting that the individual quantities on the right-hand sides of (2) turn out to be very ill-defined.

In this spirit, the axiomatic approaches focus directly on the observable aspects of a theory, that have an unambiguous and invariant physical meaning, and which should be computed in order to compare with experiment. They thus strive to develop analytic strategies to extract these quantities from a given theory. E.g., the particle spectrum emerges in terms of poles in renormalized correlation functions, or in terms of the spectrum of the time evolution operator, rather than as an input in terms of a classical action. The Haag-Ruelle scattering theory showing how the space of scattering states (and its structure as a Fock space) is intrinsically encoded, and how cross sections are obtained as asymptotic limits of correlations, was one of the first successes.

The power of the axiomatic approach resides not least in the ability to derive structural relations among elements of the theory without the need to actually compute them in a model. These relations are recognized as necessary consequences of the axioms. The most familiar examples are the PCT theorem and the Spin-Statistics theorem, which arise from functional identities among the Wightman functions due to covariance, energy positivity and locality.

Another example is the discovery ("Doplicher-Haag-Roberts theory") of the coherence among the intrinsic data relating to the superselection structure (charge structure). To value this approach, it is important to note that if one assumes (as one usually does) the presence of unobservable charged fields in a theory, these will typically "create" charged states from the vacuum state $\Omega$. As a specific example,

$$
\Psi=\psi(f) \Omega
$$

is an (electrically charged) fermionic state if $\psi(f)$ is an (electrically charged) Fermi field smeared with some function $f$. These states cannot be created by observable fields such as those in eq. (2), and their charge can be distinguished by looking at suitable characteristics of the state functional 


$$
A \mapsto \omega_{\Psi}(A) \equiv(\Psi, A \Psi)
$$

as the local observables $A$ vary, e.g., when the charge operator $Q$ is approximated by integrals over the charge density $j^{0}$. States of different charge belong to inequivalent representations (superselection sectors) of the observables. The DHR theory provides the means to study charged sectors intrinsically, i.e. without the assumption of charged fields creating them.

More recently, the DHR theory culminated in the proof ("DoplicherRoberts reconstruction") that the observables along with their charged representations in fact determine an algebra of charged unobservable fields transforming under a global symmetry group, which create charged sectors from the vacuum and among which the observables are the invariants under the symmetry [4]. Indeed, the presence of Fermi fields, although these do not correspond to observable quantities, can be inferred (and their conventional use can be justified) from the existence of fermionic representations of the bosonic fields of the theory.

At least the relevance of global symmetry has thus been derived from the physical principles of QFT. At the same time, the way how geometric properties of spacetime enter this analysis shows clearly why the analogous conclusion fails in low dimensional QFT, opening the way to a much broader symmetry concept beyond global symmetry groups.

In realistic models of QFT, the most important symmetry concept is that of local gauge groups, to which we devote a section of its own below. Unfortunately, local gauge symmetry is not covered by the DHR theory.

Axiomatic approaches also allow to investigate the infrared problem of theories containing electromagnetism. The infrared problem is due to the fact that the mathematical description of particle states as eigenstates of the mass operator

$$
P_{\mu} P^{\mu} \Psi=m^{2} \Psi
$$

(which is the starting point of the Haag-Ruelle scattering theory) cannot be used for particles which carry an electric charge. It was proven under very general conditions [5] that electrically charged sectors contain no eigenstates of the mass operator. Instead one may use so called particle weights which share many properties with particle states but are not normalizable [6].

A more pragmatic way out is the artificial introduction of a photon mass as a regulator. One computes the cross sections in the auxiliary theory and takes the limit of vanishing photon mass for suitable inclusive cross sections (where "soft" photons, i.e. photons below an arbitrary small, but finite energy in the final state are not counted) at the very end. On the conceptual level, this method involves an exchange of limits. Namely, scattering theory in the sense of Haag and Ruelle amounts to look at distances which are large compared to the Compton wavelengths of the particles. The physically relevant limit for scattering of electrically charged particles should therefore be to perform 
first the limit for the photon mass and then to go to large distances. As was emphasized by Steinmann [7], it is doubtful whether the limits may be exchanged.

The section about axiomatic approaches should not be concluded without the remark that the complete construction of models fulfilling all required principles has been achieved with methods described in Sect. 6 below, although presently only in two and three dimensional spacetime (polynomial self-interactions of scalar fields, Yukawa interactions with Fermi fields).

Low-dimensional models are of interest as testing grounds for the algebraic methods and concepts of axiomatic approaches, and to explore the leeway left by the fundamental principles. Apart from that, since string theory can in some respect be regarded as (a ten-dimensional "target space re-interpretation" of) a conformal quantum field theory in two dimensions, the exact control available for a wealth of these models could thus indirectly provide insight into higher dimensional physics.

Conformally invariant theories in two dimensions have been constructed rigorously (and partially classified [8]) by methods of operator algebras, especially the theory of finite index subfactors [9]. It is here crucial that a "germ" of the theory is given, such as the subtheory of the stress-energy tensor field, and is verified to share certain algebraic features. Then any local and covariant QFT which contains this subtheory is strongly constrained, and can be constructed from certain data associated with the subtheory. Even if the "germ" (as is usually the case) can be realized as a subtheory of some auxiliary free field theory, the ambient theories thus constructed cannot be regarded as free theories, i.e., they "extend the subtheory in a completely different direction".

Quite recently, a novel scheme for the construction of quantum field theories has been developped in a genuinely operator algebraic approach, which is not based on quantum fields and some classical counterpart, but on the relation between the localization of quantum observables and their interpretation in terms of scattering states. As a consequence of the phenomenon of vacuum polarization, this relation is subtle since interacting local fields can never create pure one-particle states from the vacuum. The basic new idea stems from modular theory (see below) by which geometric properties such as localization in causally independent regions and the action of Poincaré transformations can be coded into "modular data" of suitable algebras.

Although this is not the place to introduce modular theory [10] to a general audience, we wish to add a rough explanation. There is a mathematical theorem that the pair of a von Neumann algebra and a (cyclic and separating) Hilbert space vector determine an associated group of unitaries and an antiunitary involution, the "modular data", which have powerful algebraic and spectral properties. In the case of algebras of covariant quantum observables localized in a wedge region (any Poincaré transform of the region $|c t|<x_{1}$ ) and the vacuum vector, these properties allow to identify the modular data with a subgroup of the Poincaré group and the PCT conjugation. The joint 
data for several such wedge algebras generate the unitary representation of the full Poincaré group. Exploiting this algebraic coding of geometry in the opposite direction, it is in fact possible to construct a QFT by specifying a distinguished vector in a Hilbert space and a small number of von Neumann algebras, provided these are in a suitable "relative modular position" to each other to warrant the necessary relations among their modular data to generate the Poincaré group and ensure local commutativity and energy positivity.

This opens an entirely new road for the non-perturbative construction of quantum field theory models [11]. As an example in two spacetime dimensions, algebras of putative observables localized in spacetime wedges can be constructed in terms of one-particle states. Observables with bounded localization are then obtained by taking intersections of wedge algebras. That this road indeed leads to the desired construction of interacting theories with a complete interpretation in terms of asymptotic particle states, has been established [12] for a large class of models with factorizing scattering matrices.

\section{The Gauge Principle}

It happens very often that complicated structures can be more easily accessed by introducing redundant quantities. The extraction of the relevant information then requires a notion of equivalence. In fundamental physics it is the notion of a local interaction which forces the introduction of redundant structures. To ensure that the observable quantities do not influence each other at a distance, one wants to describe their dynamics by field equations which involve only quantities at the same point. But it turns out that this is possible only by introducing auxiliary quantities, such as gauge potentials in electrodynamics. This difficulty already exists in classical field theory, and it complicates considerably the structure of classical general relativity.

Classical gauge theories describe the interaction of gauge fields (understood as connections of some principal bundle) and matter fields (described as sections in associated vector bundles). The interaction is formulated in terms of covariant derivatives and curvatures. (In this way, the rather marginal gauge symmetry of Maxwell's electrodynamics is turned into a paradigmatic symmetry principle determining the structure of interactions.) The combination

$$
D_{\mu}=\partial_{\mu}+i e A_{\mu}
$$

providing the coupling between the fields in (1) is a covariant derivative which ensures that the equation is invariant under the gauge transformation

$$
\begin{aligned}
\psi(x) & \mapsto \mathrm{e}^{i e \alpha(x)} \psi(x) \\
A_{\mu}(x) & \mapsto A_{\mu}(x)-\partial_{\mu} \alpha(x),
\end{aligned}
$$

that is, $D_{\mu} \psi$ transforms in the same way as $\psi$ itself. The field strength tensor $F_{\mu \nu}$ in (2) is obtained through the commutator of two covariant derivatives, i.e. geometrically speaking, the curvature. 
The presence of this group of automorphisms (7) of the bundle (gauge transformations) makes the description redundant, and only the space of orbits under the automorphism group corresponds to the relevant information.

In quantum field theory, the very concept of gauge theories is strictly speaking not well defined, because of the singular character of pointlike localized quantities. These singularities are absent in the lattice approximation (see Sect. 6). There matter fields are attached to the vertices, and gauge fields are as parallel transporters attached to the links.

In perturbation theory (see Sect. 5) additional auxiliary structure has to be invoked in order to be able to use the canonical formalism. Namely, the Cauchy problem in gauge theories is not well posed because of the ambiguities associated with time dependent gauge transformations. Therefore one has to introduce a gauge fixing term in the Lagrangean which makes the Cauchy problem well posed, and so called "ghost and antighost" fields which interact with the gauge field in such a way that the classical theory is equivalent to the original gauge theory. This auxiliary theory is quantized on a "kinematical Hilbert space" $\mathcal{H}$ which is not positive definite. The observables of the theory are then defined as the cohomology of the BRST transformation $s$ which is an infinitesimal symmetry of the theory with $s^{2}=0$ (see, e.g., [13]). More precisely, $s$ is implemented as a graded derivation by a charge operator $q$ such that $q^{2}=0$, the observables are those local operators that commute with $q$ :

$$
q A=A q,
$$

physical states are those annihilated by it:

$$
q \Psi_{\text {phys }}=0,
$$

and two physical states are equivalent if they differ by a state in the image of it:

$$
\Psi_{1}-\Psi_{2} \in q \mathcal{H} .
$$

The BRST method ensures that the equivalence classes of physical states form a positive-definite Hilbert space

$$
\mathcal{H}_{\text {phys }}=\operatorname{Ker} q / \operatorname{Im} q,
$$

and the observables are well-defined operators on $\mathcal{H}_{\text {phys }}$.

We will see in Sect. 5 that within perturbation theory, BRST gauge theories are distinguished by their good behaviour under renormalization.

It is not clear how the gauge principle should enter the axiomatic formulations. These approaches focus on the observables of a quantum system, while gauge fields are per se unobservable. Put differently, one should ask the question which observable features tell us that a QFT is a gauge theory. In the abelian case, there is of course the characteristic long-range nature of Gauss' 
law, but there is no obvious equivalent in the nonabelian case, i.e. when $\psi$ in (7) is replaced by a multiplet and the phase factor $\mathrm{e}^{i e \alpha(x)}$ by a unitary matrix. Could there be, in principle, an alternative description of, say, QCD without gauge symmetry?

There are of course experimental hints towards the color symmetry, ranging from particle spectroscopy over total cross section enhancement factors to "jets" in high-energy scattering. In algebraic QFT, the counterpart of these observations is the analysis of the global charge structure of a theory, i.e. the structure of the space of states.

The DHR theory of superselection sectors is precisely an analysis of the charge structure entirely in terms of the algebra of observables. As we have seen, it leads to the derivation of a symmetry principle from the fundamental principles of QFT (see Sect. 2), but the result pertains to global symmetries only. The case of local gauge symmetries is still open. Yet, a local gauge theory without confinement should possess charged states in nontrivial representations of the gauge group. If the theory has confinement, but is asymptotically free, then its gauge group should become visible through the charge structure of an appropriate short-distance limit of the observables [1]. It is therefore expected that gauge symmetry, if it is present, is not an artefact of the perturbative description but an intrinsic property coded in algebraic relations among observables.

\section{The Field Concept}

It is the irony of Quantum Field Theory that the very notion of a "quantum field" is not at all obvious. The field concept has been developped in classical physics as a means to replace the "action at a distance" by perfectly local interactions, mediated by the propagating field. Classical fields, such as the electromagnetic fields, can be observed and measured locally. On the other hand, in quantum field theory one usually interprets measurements in terms of particles. The fields used in the theory for the prediction of counting rates, appear as (very useful, undoubtedly) theoretical constructs, imported from the classical theory. But what is their actual status in reality?

The conventional particle interpretation requires that a given state behaves like a multi-particle state at asymptotic times. A closer look shows that this feature may be expected only in certain circumstances, say, in a translationally invariant theory in states close to the vacuum. Once one leaves these situations, neither the concept of a vacuum (ground state of the energy) nor that of particles (eigenstates of the mass operator) keep a distinguished meaning, as may be exemplified by the occurrence of Hawking radiation, by the difficulties of a notion of particles in thermal states, and last not least, in the infrared problem. 
The field concept, on the other hand, keeps perfect sense in all known cases. Fields may be understood, generally speaking, as means to interpret quantum theoretical objects in geometrical terms. In Minkowski space, they may assume the form of distributions whose values are affiliated to the algebras of local observables and which transform covariantly under Poincaré transformations. Here, the test function $f$ plays the role of a "tag" which keeps track of the localization of the associated field operator $\varphi(f)$. In a generally covariant framework (see Subsect. 8.1), fields can be viewed abstractly as natural transformations from the geometrically defined functor which associates test function spaces to spacetime manifolds, to the functor which associates to every spacetime its algebra of local observables [14].

On the mathematical side, the field concept leads to hard problems in the quantum theory. They are due to the quantum fluctuations of localized observables which diverge in the limit of pointlike localization. But in perturbation theory as well as in algebraic quantum field theory one has learned to deal with these problems, the most difficult aspect being the replacement of ill-defined pointwise products by the operator product expansion.

In free field theory on Minkowski space, one associates to every particle a field which satisfies the field equation. While in this case, the use of the term "particle" for the associated field is perfectly adequate, the analogous practice for fields which appear in the classical equation of motion of interacting field theory is justified only in special cases. It may happen (this seems to be the case in asymptotically free theories) that in a short distance limit, the analogy to the particle-field correspondence of free field theory becomes meaningful. In theories which become free in the infrared limit, a similar phenomenon happens at large distances; then the scattering data can be directly interpreted in terms of these distinguished fields.

In general, however, besides the observable fields one uses a whole zoo of auxiliary fields which serve as a tool for formulating the theory as a quantization of a classical Lagrangean field theory. Such a formulation may not always exist nor must it be unique. In the functional ("path integral") approach to QFT, such auxiliary fields (which are not coupled to external sources) may be regarded as mere integration variables. The most powerful functional techniques involve deliberate changes in such variables (introduction of "ghost fields", BRST transformations, or the renormalization program by successive integration over different energy scales). While this is by far the most successful way to construct models, at least in the sense of perturbation theory, the intrinsic physical significance of these auxiliary fields is unclear, and it would be misleading to think of them in terms of particles in a similar way as discussed before.

The delicacy of the field concept in Quantum Theory, contrasted with the clarity of the classical field concept, may be just one aspect of the more fundamental question: Is a quantum theory necessarily the quantization of a 
classical theory? Does it always have a classical limit (think of QCD, for the sake of definiteness), and can it be reconstructed from its classical limit?

\section{The Perturbative Approach to QFT}

The main approximative schemes for relativistic QFT are Perturbation Theory (or other expansions like the $1 / N$ approximation), and lattice approximations of Euclidean functional integrals. All these approximations of QFT are based on the idea of "quantization of a classical field theory". Perturbation theory proceeds by producing a formal power series expansion in a coupling constant, hoped to be asymptotic to a QFT yet to be constructed, and therefore requires weak couplings; lattice approaches can in principle also treat strongly coupled regimes, using cluster expansions or Monte Carlo simulations; although numerical simulations of lattice QFT are limited to rather coarse lattices, aspects of the continuum and infinite volume limits can be studied. As far as comparisons are possible, there seems to be little doubt about the basic consistency among different approaches.

Our discussion in this section will mainly pertain to Perturbation Theory. This is a general scheme a priori applicable to any Lagrangean with a "free" and an "interaction" part. Characteristic limitations to the scheme arise, however, through various sources:

First of all, there is the need to "renormalize" the single terms of the perturbative expansion. This is the procedure to fix the parameters of the theory to their physical values, thereby also avoiding any infinities that occur if one proceeds in the traditional way using "bare" parameters. One must demand that renormalization can be achieved without the introduction of infinitely many new parameters which would jeopardize the predictive power of the theory. This necessity restricts the admissible form of the interaction Lagrangean. Provided the polynomial order in the fields is limited (depending on the spacetime dimension, and on the spins of the fields involved), a simple "power counting" argument (controlling the behaviour of potentially divergent terms in terms of the momentum dependence of propagators and interactions) ensures renormalizability. For spins larger than 1, there are no interactions in four dimensions which are renormalizable by power counting. (This fact also prevents the direct incorporation of gravitational fields into the perturbative scheme.) In the presence of additional symmetries which ensure systematic cancellations of divergent terms, renormalizability might be shown in spite of a failure of the power counting criterium (but in supersymmetric perturbative gravity the desired effect seems to fail beyond the first few lowest orders).

For the theory of elementary particles, experiments have revealed the prime relevance of vector (spin 1) couplings, starting with parity violation in the weak interaction which could be explained by $V-A$ but not by scalar and tensor couplings. The idea that vector couplings are mediated by vector 
fields lies at the basis of the Standard Model. For interactions involving massless vector fields, however, there is a conflict between locality, covariance, and Hilbert space positivity, while massive vector fields do not possess couplings which are renormalizable by power counting. This is due to the fact that the necessary decoupling of modes which otherwise would give rise to states of negative norm, changes the large-momentum behaviour of the propagator.

The only successful way to incorporate vector fields into a perturbative QFT is to treat them as gauge fields, with couplings which are necessarily gauge couplings (see Sect. 3). Thus, the gauge principle imposes itself through the inherent limitations of the perturbative scheme [15]. However, it brings about several new problems which have to be solved in turn: the unphysical degrees of freedom can be eliminated by cohomological methods ("BRST theory", see Sect. 3) which at the same time can be used to systematically control the preservation of gauge invariance. While gauge invariance forbids the introduction of explicit mass terms for the vector fields, masses can be generated by coupling to a Higgs field with "spontaneous symmetry breakdown". That this can indeed be done in a way which keeps the theory renormalizable in spite of the bad power counting behaviour of massive propagators, is one of the great achievements of the perturbative Standard Model.

In the process of renormalization there may appear "anomalies" which break symmetries present in the classical theory. While anomalies per se are not problematic (and may even be phenomenologically desirable), anomalies of the gauge symmetry will spoil the renormalizability. Their absence has therefore to be imposed as a consistency condition. In chiral gauge theories, it can be achieved by a suitable choice of representations of the gauge group (particle multiplets).

The circumstance that the "cascade of problems" outlined in the preceding paragraph can in fact be consistently overcome within the setting of perturbative QFT, and in excellent agreement with the phenomenology of High Energy Physics, gives enormous confidence in the basic correctness of the approach. The Standard Model precisely exhausts the leeway admitted by the perturbative approach.

Besides the renormalization problems caused by ultraviolet singularities, perturbative QFT has infrared problems, when the free theory used as the starting point contains massless particles. In Quantum Electrodynamics (QED), the infrared problem can be traced to the computational use of particles with sharp masses which is illegitimate in the presence of massless particles (see Sect. 5).

A more severe kind of infrared problem arises in theories like QCD; here it is due to the fact that the fields (quarks and massless gluons) do not correspond to the massive particles (hadrons) presumably described by the full, non-perturbative theory. A fully consistent solution of these problems, i.e. the confinement of hadronic constituents, can therefore not be expected in a perturbative treatment. If the confinement problem can be addressed at all, then 
only by non-perturbative methods (see the next section). However, effects like the deviations from naive scaling of hadronic structure functions have been successfully predicted by perturbative methods.

The infrared problems of perturbation theory may be circumvented by the use of interactions which are switched off outside some compact region of spacetime. This leads to the concept of causal perturbation theory which was developed by Epstein and Glaser [16] on the basis of previous ideas of Stückelberg and Bogoliubov. This approach is crucial for a consistent treatment of quantum field theory on curved spacetimes. On Minkowski space it allows a perturbative construction of the algebra of observables. The infrared problem then is the physical question on the states of the theory, such as the existence of a ground state, the particle spectrum, thermal states and so on.

Whether one considers the rationale for the gauge principle in the Standard model outlined above (see also Sect. 3) to be logically cogent, depends on the implicit expectations one imposes on the formal structure of a QFT. In any case, the Standard Model is by no means uniquely determined by these constraints. QED and QCD are completely self-consistent subtheories (that is on the level of a formal perturbative expansion); the subtheory of electroweak interactions is consistent provided the gauge anomalies are eliminated by suitable charged multiplets. The gauge groups themselves may be considered as free parameters of a model, as long as anomaly cancellation is possible.

The possibility of Grand Unification and/or Supersymmetric Extensions is an aesthetic feature of the Standard Model, for which, however, there is no fundamental physical need, nor is it required for reasons of mathematical consistency. QFT alone presumably cannot answer the question why there are so many "accidental" free parameters (notably the mass matrices, or Yukawa couplings, according to the point of view) in the theory of fundamental interactions.

To conclude this section, we should point out that, as far as model building is concerned, the limitation to renormalizable interactions might be too narrow. There are perturbatively non-renormalizable model theories in which nontrivial fixed points have been established, meaning that the theories are non-perturbatively renormalizable [19].

\section{The Constructive Approach to QFT}

In spite of its tremendous numerical success, the perturbative scheme to evaluate QFT approximately suffers from a severe defect: it provides answers only in the form of formal, most likely divergent power series. The usual answer to this is that the series is an asymptotic expansion. But aside from the problem where to truncate the series in order to convert the formal power series into numbers, there is the fundamental question: asymptotic to what? There are well-known cases (such as the so-called $\Phi_{4}^{4}$ theory of a self-interacting scalar 
field $\Phi$ in four spacetime dimensions) in which the perturbation expansion, according to the accumulated knowledge, is not an asymptotic expansion to any QFT and it may very well be that the most successful of all QFT's, Quantum Electrodynamics, also suffers from this disease.

The axiomatic approach, on the other hand, does not answer the question whether the axioms are not empty, i.e. whether any nontrivial QFT's satisfy them.

The constructive approach is in principle addressing both of these problems. On the one hand it attempts to show that the axiomatic framework of QFT is not empty, by mathematically constructing concrete nontrivial examples satisfying these axioms, and on the other hand it provides nonperturbative approximation schemes that are intimately related to the attempted mathematical constructions; the prime example are the lattice approximations to QFT's. Even where the goal of a mathematical construction of models satisfying all the axioms is not (yet) attained, this kind of approximative scheme differs in a fundamental way from the formal perturbative expansions: it produces approximate numbers which, if all goes right, converge to a limit that would be the desired construction.

The constructive approach (see for instance [17]) is based on a modification and generalization of Feynman's "sum over histories". The main modification is the transition form the indefinite Lorentz metric of Minkowski spacetime to a Euclidean metric; the return to the physical Lorentzian metric is expected to be manageable via the so-called Osterwalder-Schrader reconstruction [2] (see Sect. 2). The approach starts from a classical field theory, with dynamics specified by a Lagrangean. Formally one then procedes by writing an ill-defined functional integral over all field configurations, weighted with a density given in terms of the classical action $S=\int \mathcal{L} d x$ depending on some fields collectively denoted by $\Phi$; the expectation value of an "observable" $\mathcal{O}[\Phi]$ (a suitable function of the fields) would be given by

$$
\langle\mathcal{O}\rangle=\frac{1}{Z} \int \mathcal{D} \Phi \mathcal{O}[\Phi] e^{-S[\Phi]} .
$$

Here the symbol $\mathcal{D} \Phi$ is supposed to indicate a (nonexisting) Lebesgue measure over the set of all field configurations $\Phi$ and $Z$ a normalization constant.

To make mathematical sense of this, the theory first has to be "regularized" by introducing a finite spacetime volume and deleting or suppressing high frequencies (by an "ultraviolet cutoff"). The job of the constructive field theorist then consists of controlling the two limits of infinite volume ("thermodynamic limit") and restoring the high frequencies ("ultraviolet limit") by removing the cutoff; the latter can only be done if the parameters of the Lagrangean (and the observables of the theory) are made cutoff dependent in a suitable way - this procedure is the non-perturbative version of renormalization.

The constructive program has been completed only in spacetime dimensions less than four, but at least in these unrealistic cases it has shown that 
axiom systems such as Wightman's are not vacuous for interacting theories. In these low-dimensional cases it has also given a justification to the perturbative expansion by showing that it produces indeed an asymptotic expansion to the constructed QFT's.

A particularly useful way of introducing an ultraviolet cutoff consists in replacing the spacetime continuum by a discrete structure, a lattice. Together with the introduction of a finite spacetime volume one thereby reduces quantum field theory to a finite dimensional "integral" (the quotation marks indicate that this "integral" is just some linear form for the fermionic degrees of freedom). In other words, QFT has been reduced to quadratures. The advantage of this is that QFT thereby becomes amenable to numerical evaluation; there is a whole industry of lattice field theory exploiting this fact, most notably in approximately evaluating the theory of strong interactions, QCD.

But the lattice approach is very important also for more fundamental reasons: it is the only known constructive approach to a non-perturbative definition of gauge field theories, which are the basis of the Standard Model. The constructive approach and the numerical procedures to extract infinite volume and continuum information from finite lattices are closely parallel:

Typically a lattice model produces its own dynamically generated scale $\xi$ ("correlation length") which, unlike the lattice spacing, has a physical meaning. It may be defined - after the thermodynamic limit has been taken - by the exponential decay rate of suitable correlation functions, such as

$$
\xi=-\lim _{n \rightarrow \infty} \frac{1}{|n|} \ln \langle\Phi(0) \Phi(n)\rangle,
$$

where $\Phi(\cdot)$ stands for a field of the lattice theory and $n$ is a tupel of integers labeling lattice points.

In a finite volume version, finite volume effects disappear exponentially fast, like $\exp (-L / \xi)$, with the size $L$ of the volume. The thermodynamic limit can then be controlled numerically and often also mathematically, borrowing techniques from classical Statistical Mechanics.

The next step is to identify the dimensionless number $\xi$ with a physical standard of length (e.g., some appropriate Compton wave length, say $1 \mathrm{fm}$ ), such that $\xi$ lattice spacings equal $1 \mathrm{fm}$. The lattice points can then be relabeled by $x_{i}=\left(n_{i} / \xi\right)$ fm where the coordinates $x_{i}$ have now acquired the dimension of length. Taking the lattice spacing to zero (i.e. taking the continuum limit) then amounts to sending the correlation length to infinity while keeping $x_{i}$ fixed. The $n$-point correlation functions of a field in the continuum should therefore be defined as limits of the form

$$
\left\langle\varphi\left(x_{1}\right) \ldots \varphi\left(x_{n}\right)\right\rangle=\lim _{\xi \rightarrow \infty}\left\langle\Phi\left(\left[x_{1}\right] \xi\right) \ldots \Phi\left(\left[x_{n}\right] \xi\right)\right\rangle Z(\xi)^{-\frac{n}{2}}
$$

where $x=[x] \mathrm{fm}$, and $\varphi(x)$ is the resulting continuum quantum field.

So the continuum limit requires to drive the parameters of the system (such as the coupling constants) to a point of divergent correlation length, 
i.e. a critical point in the language of Statistical Mechanics. $Z(\xi)$ is a "field strength renormalization" needed to prevent the limit from being 0 .

This procedure makes it clear that the lattice spacing is a derived dynamical quantity proportional to $1 / \xi$, not something to be specified beforehand. The inverse of the correlation length in the chosen physical units is the mass gap of the theory in physical units. The procedure of choosing the dynamically generated scale as the standard of length or mass leads generally to a phenomenon usually attributed to special features of perturbation theory: "dimensional transmutation". Let us explain this in a simple case, QCD with massless quarks: the only parameter of the lattice theory is the gauge coupling; since we find the continuum limit at the (presumably unique) critical point, this ceases to be an adjustable parameter. Instead we obtain a free scale parameter by the freedom of choosing a certain multiple of the correlation length as the standard of length (or a certain multiple of the inverse correlation length as the standard of mass). So we have traded a dimensionless parameter (the coupling constant) for a parameter with dimensions of a mass (e.g., the mass of the lightest particle).

Quite generally the particle spectrum of any QFT is extracted by looking at exponential decay rates of suitable correlation functions; when applied to QCD the lattice approach has been reasonably successful in reproducing the observed spectrum of baryons and mesons. It has also been successfully extended to the computation of weak decay matrix elements of hadrons. All this gives us confidence that QCD is indeed an appropriate description of the strong interactions.

On the constructive side, the success with gauge theories in four dimensions has been much more modest, even though some impressive mathematical work towards control of the continuum limit has been done by Balaban [18].

\section{Effective quantum field theories}

In applications one often encounters the term "effective field theory". We can distinguish three different meanings:

(1) the result of an exact Renormalization Group (RG) transformation applied to a quantum field theory in the sense discussed before,

(2) an approximate quantum field theory that is supposed to give a good approximation to a certain assumed QFT,

(3) a phenomenological theory that is not to be taken seriously beyond a certain energy; in this case it does not matter if the theory arises from a bona fide QFT by some approximation or by integrating out high momentum modes.

The notion (1) is at least conceptually very clear. The idea is to start with an already constructed well-defined quantum field theory and then to apply an exact "Renormalization Group step". This means that one performs 
the part of the functional integral (which has been made well-defined before) corresponding to the "hard" (i.e. high momentum, fast varying) part of the fields, formally

$$
\exp \left(-S_{\text {eff }}\left[\Phi_{\text {soft }}\right]\right)=\frac{1}{Z} \int \mathcal{D} \Phi_{\text {hard }} \exp \left(-S_{\text {eff }}\left[\Phi_{\text {soft }}+\Phi_{\text {hard }}\right]\right) .
$$

and also performs some rescalings of fields and spacetime variables. The combination of the integration in (15) and this rescaling constitutes one Renormalization Group step. The resulting "effective theory" describes exactly the same physics as the original full theory when applied to the soft (low momentum, slowly varying) degrees of freedom. It is clear that this may be "effective", but it is not efficient because it requires control of the full theory before one can even start.

Of course the RG step sketched above can be iterated; thereby one generates the semigroup usually called Renormalization Group.

A more useful variation of the RG idea is used in Constructive Quantum Field Theory (see for instance $[18,19]$ ). Here one starts with a regularized version of the theory, defined with a high momentum cutoff; one then performs a number of RG steps as indicated above until one reaches a predefined "physical scale" leading to an effective low energy theory still depending on the cutoff. In the final step one attempts to show that the low energy effective theory has a limit as the cutoff is removed; this requires adjusting the parameters of the starting "bare action" such that the effect of the increasing number of successive Renormalization Group steps is essentially compensated.

The notion (2) is widely used to describe the low energy physics of QCD (assumed to exist as a well-defined Quantum Field Theory even though this has not been shown so far). Specific examples are:

- "Effective Chiral Theory" [20] to describe the interactions of the light pseudoscalar mesons,

- "Heavy Quark Effective Theory" (HQET) [21], in which the effect of the heavy (charmed, bottom and top) quarks is treated by expanding around the limit where their masses are infinite,

- "Nonrelativistic QCD" (NRQCD) [22, 23] used in particular for bound state problems of heavy quarks.

For an overview over various applications of these ideas see [24].

Examples for notion (3) are the old Fermi theory of weak interactions (before the electro-weak part of the Standard Model was known). A more modern example is presumably the Standard Model itself, because it contains the scalar self-interacting Higgs field which suffers from the presumed triviality of $\Phi_{4}^{4}$ theories; the same applies to any other model involving Higgs fields. One often finds the words "something is only an effective theory"; this expresses 
the fact that the author(s) do not want to claim that their model corresponds to a true quantum field theory.

\section{Gravity}

Given the state of affairs for the Standard Model of elementary particles, being comfortably well described by Quantum Field Theory as outlined in the previous sections, the "missing link" in our present conception of fundamental physics is the incorporation of the gravitational interaction into quantum physics (or vice versa).

For a review of classical Gravity, we refer to the contribution by Ehlers to this volume. Empirically, Gravity is a theory valid at macroscopic scales only, and it is well known that, if extrapolated to very small scales (the Planck length), it becomes substantially incompatible with the quantum uncertainty principle ("quantum energy fluctuations forming virtual black holes"). This suggests that at small scales Gravity needs modification, although one might as well argue conversely, that at small scales Gravity modifies Quantum Theory (by acting as a physical regulator for the UV problems of QFT, or possibly in a much more fundamental manner). The truth is not known, and one might expect that neither Quantum Theory nor Gravity will "survive" unaffected in the ultimate theory.

Empirical evidence for this case is of course extremely poor due to the smallness of the Planck length. The most promising candidate for empirical evidence about effects of Quantum Gravity are astronomical observations of matter falling into supermassive black holes, or cosmological remnants of the very early universe. On the theoretical side, it is generally expected that black hole physics (Hawking radiation and Bekenstein entropy) represents the crucial point of contact. It appears very encouraging that both major approaches (String Theory and Canonical Quantum Gravity, see below), in spite of their great diversity, make more or less the same predictions on this issue. But it should be kept in mind that also Hawking radiation of black holes is far from being experimentally accessible.

The attempt to incorporate the gravitational interaction into Quantum Theory raises severe conceptual difficulties. Classical gravity being a field theory, QFT is expected to be the proper framework; but QFT takes for granted some fixed background spacetime determining the causal structure, as one of its very foundations, while spacetime should be a dynamical agent in gravity theory. This argument alone does not preclude the logical possibility of perturbative quantization of gravity around a fixed background, but on the other hand, the failure of all attempts so far which split the metric into a classical background part and a dynamical quantum part (cf. Sect. 2), should not be considered as a complete surprise, or as a testimony against QFT. 
On the other hand, the existing arguments against the quantization of gravity within a conventional QFT framework are not entirely conclusive. They are based on the most simple notion of renormalizability which demands that the renormalization flow closes within a finite space of polynomial couplings, thus giving rise to the limitation by power counting. It is conceivable, and there are indications that something in this way actually occurs [25], that a renormalization flow closes within a finite space of suitable nonpolynomial Lagrangeans (which are present in classical Einstein gravity anyway). In this case, the renormalized theory also would contain only finitely many free parameters, and would have the same predictive power as a theory with polynomial interactions.

Taking the geometrical meaning of gravitational fields seriously, it is clear that the framework of QFT has to be substantially enlarged in order to accomodate a quantum theory of Gravity. It is an open question whether this can be done by formal analogies between diffeomorphism invariance and gauge symmetry.

\subsection{QFT on gravitational background spacetime}

An intermediate step on the way towards a theory of Quantum Gravity is a semiclassical treatment, where "matter" quantum fields are defined on classical curved spacetimes. This situation brings along severe technical and conceptual problems, since crucial tools of quantum field theory in flat spacetime (energy-momentum conservation, Fourier transformation and analyticity, Wick rotation, particle interpretation of asymptotic scattering states) are no longer available due to the lack of spacetime symmetries.

Considerable progress in this direction has been made notably concerning the problem of the absence of a distinguished ground state (the vacuum). In globally hyperbolic spacetimes, the ground state can be substituted by a class of states (Hadamard states) which guarantee the same stability properties of quantum fields, and allow for a similar general set-up of causal perturbation theory as in flat space [26]. Of crucial importance is the incorporation of the principle of general covariance. It is realized as a covariant functor which associates to every globally hyperbolic spacetime its algebra of observables and which maps isometric embeddings of spacetimes to homomorphic embeddings of algebras. The interpretation of the theory is done in terms of covariant fields, which are mathematically defined as natural transformations from a geometrically defined functor which associates to every spacetime its test function space to the functor describing the quantum field theory [14].

One may include into the set of quantum fields also the fluctuations of the metric field. One then has to impose the consistency condition that the result does not depend on the chosen split of the metric into a background field and a fluctuation field (this is essentially Einstein's equation in quantum field theory). One may hope to obtain in this way reliable information on the "back 
reaction" of the energy of the quantum matter on the background. It remains, however, the bad power counting behaviour of quantum gravity which might point to limitations of the perturbative approach.

\subsection{Non-commutative spacetime}

Taking into account the expectation that localization should be an operational concept which at very small scales is limited by the interference between quantum and gravitational effects, models of non-commutative spacetimes have been formulated which exhibit an intrinsic localization uncertainty. While these are definitely not more than crude models, in which gravity is not itself present but just motivates the localization uncertainty, it could be established that they are compatible with Quantum Field Theory; contrary to widespread hopes, however, the quantum structure of spacetime does not act as a "physical regulator" at the Planck scale for the ultraviolet behaviour of quantum field theory [27]

\subsection{Canonical Quantum Gravity}

Other approaches to Quantum Gravity focus on the purely gravitational selfinteraction. The most prominent ones, going under the name "Canonical quantum gravity", are built upon the geometric nature of classical gravity. In these approaches, the dynamics of three-dimensional (space-like) geometries is studied in a canonical framework. However, due to general covariance, the dynamics turns out to be strongly constrained, giving rise to severe complications. (See the contribution of Giulini and Kiefer to this volume.)

Within the general framework of canonical approaches, Loop Quantum Gravity (LQG) has been pursued and developped furthest as a model for the structure of quantum spacetime [28] (see also the contributions of Thiemann and of Nicolai and Peeters to this volume). It is asserted that the model can be supplemented by any kind of "conventional" matter (e.g., the Standard Model). It therefore denies every ambition towards a unified or unifying theory.

For these reasons, critical questions confronting the model with the requirements for a "true" theory of Quantum Gravity, are more or less void. As for its intrinsic consistency and mathematical control, the model meets rather high standards, consolidating and improving previous attempts of canonical quantization of Gravity.

The model predicts that geometric observables such as areas and volumes are quantized, with lowest eigenvalue spacings of the order of the Planck size. This feature appears most promising in that quantum deviations from classical geometry are derived as an output, with no classical ("background") geometry being used as an input.

On the other hand, one of the most serious flaws of LQG is the lack of understanding of its relation to gravity "as we know it", i.e. the construction 
of semiclassical states in which Einstein's General Relativity at large scales is (at least in some asymptotic sense) restored.

Another, presumably related drawback of LQG (like any other model within the canonical approach to Quantum Gravity) is that in the physical Hilbert space, once it has been constructed, the Hamiltonian vanishes. Thus, the question of the nature of "time" evolution of the quantum gravitational states is presently poorly understood.

\subsection{String Theory}

A detailed discussion of successes and problems of String Theory will be given in contributions of Louis, Mohaupt and Theisen to this volume. We will here restrain ourselves to some questions focussing on the intrinsic structure and the conceptual foundations of String Theory, which appear quite natural to ask having in mind the benefits of axiomatic approaches in the case of QFT. Even if some of our questions might appear immodest, the theory being still under construction, they should be settled in some sense before String Theory can be considered as a mature theory.

String Theory is a quantum theory naturally including gravitational degrees of freedom in a unified manner along with "conventional" matter. Gravitons and other particles arise as different "zero modes" of strings which are the fundamental objects; higher vibrational modes would correspond to undetected heavy particles (with masses far beyond accelerator energies). This fact is the prominent source of enthusiasm with the theory. (For a critical comparison of the achievements of String Theory and of Loop Quantum Gravity as candidates for the quantum theory of gravitation, see e.g., [30].)

The theory can successfully reproduce scattering cross sections for gravitons as they are expected in the lowest orders of perturbation theory with the Einstein-Hilbert action. In contrast to perturbation theory (cf. Sect. 5), the theory is believed to have a better UV behaviour due to the finite size of the string, but its alleged finiteness (or renormalizability) could not be established with the increasing understanding of higher order contributions to string theory.

On the phenomenological side, it was hoped that a unified theory including the Standard Model of elementary particles would naturally emerge as an effective theory at low (compared to the Planck scale) energies, but these hopes were considerably reduced by an enormous number of possible "string vacua", destroying the predictive power of the theory.

String theory was originally formulated in a perturbative scheme, where spacetime appears just as a classical background. The dynamics of the string moving in this background is given by a two-dimensional conformal quantum field theory (organizing its internal degrees of freedom), whose consistency requires the background to satisfy Einstein's equations. In the course of time it became clear, that a consistent formulation of string theory has to take 
into account non-perturbative structures like duality symmetries, including the need to introduce higher-dimensional objects ("branes"). The presence of these classical objects is expected to be related to the question (although still far from answering it) of the quantum nature of spacetime itself [29].

Non-perturbative formulations of string theory are in the focus of most modern developments. Yet, the mathematical structure of non-perturbative string theory and the picture of spacetime and quantum gravity which emerges, are at the present time not yet well understood beyond a huge body of heuristic imagination, based on the various duality symmetries of string theory and the "holographic principle" concerning the quantum degrees of freedom of general relativity. A most fascinating recent development is Maldacena's conjecture which states that non-perturbative string theory could be "equivalent" (in a sense involving duality) to a quantum field theory, possibly even in four dimensions. The theory which started off to supersede QFT may in the end be equivalent to a $\mathrm{QFT}$ !

As a computational scheme, String Theory is highly constrained and determined by its internal consistency. For this reason, it is often claimed to be a "unique" theory, hence it makes little sense to "axiomatize" String Theory in a similar way as Quantum Field Theory was axiomatized (Sect. 2). Nevertheless, the justification of its computational rules deserves some critical scrutiny.

The central question is: Which are the fundamental insights into the nature of physical laws (principles) that are implemented by String Theory? Is String Theory unique in doing so, or is it possibly only one consistent realization of the same principles? Accepted principles such as Quantum Uncertainty, Locality and General Relativity should be transcended by the new principles without recourse to (classical) notions outside the Theory.

An important "message" from algebraic QFT is that the intrinsic invariant structure of the quantum observables are their algebraic relations such as local commutativity, rather than their description in terms of fields. (Neither the classical action nor Feynman diagrams are intrinsic; field equations and canonical commutation relations cannot even be maintained after quantization.) The "concrete" (Hilbert space) representations of these "abstract" algebraic relations determine the physical spectrum (masses, charges).

In this spirit, one would like to identify the intrinsic elements of String Theory, and the structural relations which hold a priori among them. An intrinsic characterization would also turn claims such as the Maldacena conjecture into predictions that can be verified (or falsified).

It is generally agreed that a classical background manifold should not appear in an ultimate formulation of String Theory. This is not only because the metric is expected to fluctuate, so that it is impossible to describe its expectation values in a particular state by a classical geometry. Since spacetime structures smaller than the string size cannot be probed, and hence cannot 
have an operational meaning, String Theory is expected to produce a radically new concept of spacetime.

While String Theory is an S-matrix theory, that is, in a suitable limit it admits the computation of "on-shell" particle scattering amplitudes, "off-shell" String Field Theory has been rigorously constructed only without interactions [31]. The resulting theory may be viewed as a collection of infinitely many "ordinary" quantum fields, but their local commutativity cannot be ensured in a covariant way. The reason is that the constraints on the string degrees of freedom prevent the construction of sharply or only compactly localized observables on the physical (positive-definite) Hilbert space out of string fields defined on an indefinite space. (In view of the previous remark, this conflict with the classical spacetime concept should not come as a surprise.) With interactions, the description in terms of an infinite tower of quantum fields is expected to survive, but the structure of the interactions (string corrections to the effective action) goes beyond the framework of local Lagrangean quantum field theory. Correspondingly, String Field Theory (even in a regime where gravity can be neglected) is not expected to be a QFT in the sense of Sects. 2 or 5 .

On the other hand, String Theory exhibits a new fundamental symmetry called "duality". The Maldacena conjecture suggests that under a duality transformation, String Theory could turn into a quantum field theory. A clarification of the precise non-perturbative meaning of this conjecture is highly desirable, not least in view of the numerous and far-reaching implications drawn from it.

As an example, $T$-duality, relating vibrational and winding modes of a string, is a most characteristic symmetry of String Theory. With the help of $T$-duality one can understand how a string fails to be able to probe certain singularities of a classical background [29]. Positing duality symmetry as an abstract fundamental symmetry is a promising candidate for an intrinsic structure of the theory which can be formulated without recourse to the classical picture of a string embedded into spacetime.

As for the intrinsic texture of String Theory (assuming it to be a consistent theory), it would be desirable to understand in which sense its subtheories ("spacetime without matter", "QFT without Planck scale gravity") are separately consistent, or rather only effective theories obtained by a singular limit, which is regulated by the full theory.

While some of these questions might indeed rather reflect the authors' personal rooting in QFT (and also some lack of understanding of String Theory), we think that they are urgent enough that expert string theorists should provide answers in order to legitimate String Theory as a candidate for the Fundamental Unified Theory of all interactions. 


\section{Conclusions and Outlook}

Whether the remaining gaps in the theory are merely of technical nature, or rather signal a fundamental shortcoming of Quantum Field Theory, is not known at present, and is by many researchers not considered as the most urgent question.

Instead, the prime concern at present is the clash between Gravity and Quantum Theory, whose unification is considered as the (last) "missing link" in our conception of fundamental physics. There are promising candidate theories to achieve this ambitious goal, but none of them shares the same conceptual clarity as has been attained for Quantum Field Theory, nor are there empirical data available favouring or disfavouring either of them.

Unlike almost every historical precedent, the guiding principle at the frontiers of research in fundamental physics is therefore mainly intrinsic consistency, rather than empirical evidence. Every active researcher should be aware of the delicacy of such a situation.

It should be remarked that, while various lines of research presently pursued call basic notions such as Geometry and Symmetry into question, the basic rules of Quantum Theory are never challenged. One may be tempted to ascribe this fact to the solidity of our conceptual understanding of Quantum Physics, developped over several decades not least in the form of Quantum Field Theory. 


\section{Note to the references}

There is a long list of standard textbooks on Quantum Field Theory. The subsequent list of references leaves out most of them, as well as much of the "classical" research articles. Instead, it includes a number of less well-known articles, stressing some points which are relevant in our discussion but which do not belong to the common knowledge about quantum field theory.

\section{References}

1. D. Buchholz, R. Haag: The quest for understanding in relativistic quantum physics, J. Math. Phys. 41 (2000) 3674-3697.

2. K. Osterwalder, R. Schrader: Axioms for Euclidean Green's functions, Commun. Math. Phys. 31 (1973) 83; Commun. Math. Phys. 42 (1975) 281.

3. H. Bostelmann: Phase space properties and the short distance structure in quantum field theory, J. Math. Phys. 46 (2005) 052301.

4. S. Doplicher, J.E. Roberts: Why there is a field algebra with a compact gauge group describing the superselection structure in particle physics, Commun. Math. Phys. 131 (1990) 51-107.

5. D. Buchholz: The physical state space of quantum electrodynamics, Commun. Math. Phys. 85 (1982) 49.

6. D. Buchholz, M. Porrmann and U. Stein: Dirac versus Wigner: Towards a universal particle concept in local quantum field theory, Phys. Lett. B 267 (1991) $377-381$.

7. O. Steinmann: Perturbative Quantum Electrodynamics and Axiomatic Field theory, Springer-Verlag, Berlin etc. 2000.

8. Y. Kawahigashi and R. Longo: Classification of two-dimensional local conformal nets with $c<1$ and 2-cohomology vanishing for tensor categories, Commun. Math. Phys. 244 (2004) 63-97.

9. R. Longo and K.-H. Rehren: Nets of subfactors, Rev. Math. Phys. 7 (1995) 567-598.

10. H.-J. Borchers: On revolutionizing quantum field theory with Tomita's modular theory, J. Math. Phys. 41 (2000) 3604-3673.

11. B. Schroer and H.-W. Wiesbrock: Modular constructions of quantum field theories with interactions, Rev. Math. Phys. 12 (2000) 301;

H.-J. Borchers, D. Buchholz and B. Schroer: Polarization-free generators and the S-matrix, Commun. Math. Phys. 219 (2001) 125-140.

12. G. Lechner: An existence proof for interacting quantum field theories with a factorizing S-matrix, math-ph/0601022.

13. S. Weinberg: The quantum theory of fields, Cambridge University Press, Cambridge, 1996.

14. R. Brunetti, K. Fredenhagen and R. Verch: The generally covariant locality principle: A new paradigm for local quantum physics, Commun. Math. Phys. 237 (2003) 31-68. 
15. R. Stora: Local gauge groups in quantum field theory: perturbative gauge theories, talk given at ESI workshop "Local Quantum Physics", Vienna (1997);

D.R. Grigore: On the uniqueness of the non-Abelian gauge theories in EpsteinGlaser approach to renormalisation theory, Rom. J. Phys. 44 (1999) 853-913 [arXiv:hep-th/9806244].

M. Duetsch and B. Schroer: it Massive vector mesons and gauge theory, J. Phys. A 33 (2000) 4317.

16. H. Epstein, V. Glaser: The role of locality in perturbation theory, Ann. Inst. H. Poincaré A 19 (1973) 211.

17. J. Glimm and A. Jaffe: Quantum Physics, a Functional Integral Point of View, Springer-Verlag, Berlin etc. 1987.

18. T. Balaban: The large field renormalization operation for classical N-vector models, Commun. Math. Phys. 198 (1998) 493, and references therein.

19. K. Gawedzki and A. Kupiainen: Renormalizing the nonrenormalizable, Phys. Rev. Lett. 54 (1985) 2191; Renormalization of a nonrenormalizable quantum field theory, Nucl. Phys. B 262 (1985) 33; Gross-Neveu model through convergent perturbation expansions, Commun. Math. Phys. 102 (1985) 1.

20. J. Gasser and H. Leutwyler: Chiral perturbation theory: expansions in the mass of the strange quark, Nucl. Phys. B 250 (1985) 465; Low-energy expansion of meson form factors, Nucl. Phys. B 250 (1985) 517; $\eta \rightarrow 3 \pi$ to one loop, Nucl. Phys. B 250 (1985) 539;

21. E. Eichten and B. Hill: An effective field theory for the calculation of matrix elements involving heavy quarks, Phys. Lett. B 234 (1990) 511; Static effective field theory: 1/m corrections, Phys. Lett. B 243 (1990) 427.

22. W. E. Caswell and G. P. Lepage: Effective Lagrangians for bound state problems in QED, QCD, and other field theories, Phys. Lett. B 167 (1986) 437.

23. G. T. Bodwin, E. Braaten and G. P. Lepage: Rigorous QCD analysis of inclusive annihilation and production of heavy quarkonium, Phys. Rev. D 51 (1995) 1125; erratum Phys. Rev. D 55 (1997) 5853.

24. A. Pich: Effective field theory, Les Houches lectures 1997, hep-ph/9806303.

25. O. Lauscher, M. Reuter: Ultraviolet fixed point and generalized flow equation of quantum gravity, Phys. Rev. D 65 (2001) 025013;

M. Niedermaier: On the renormalization of truncated quantum Einstein gravity, JHEP 12 (2002) 066.

26. S. Hollands and R.M. Wald: On the renormalization group in curved spacetime, Commun. Math. Phys. 237 (2003) 123-160.

27. D. Bahns, S. Doplicher, K. Fredenhagen and G. Piacitelli: On the unitarity problem in space/time noncommutative theories, Phys. Lett. B 533 (2002) 178181.

28. A. Ashtekar: Gravity and the quantum, New J. Phys. 7 (2005) 198 [grqc/0410054].

29. G. T. Horowitz: Spacetime in string theory, New J. Phys. 7 (2005) 201 [grqc/0410049].

30. L. Smolin: How far are we from the quantum theory of gravity?, hep-th/0303185

31. J. Dimock: Locality in free string field theory, J. Math. Phys. 41 (2000) 40; Ann. H. Poinc. 3 (2002) 613. 